\title{
Design and Control of Dual-Arm Cooperative Manipulator using Speech Commands
}

\author{
N. Saravanan, R. Sivaramakrishnan
}

\begin{abstract}
Cooperative manipulators are among the subject of interest in the scientific community for the last few years. Here an overview of the design and control of such cooperative manipulators using Speech Commands in English, Hindi, and Tamil is discussed. Here we choose two identical Robot arms from lynxmotion, and both manipulators move in conjunction with one another to achieve more payload while grasping or handling the object by the end effector. The simultaneous control of identical robot manipulators could be performed by pronouncing simple speech commands by the end user using a smartphone, which then is converted into text format using a speech recognition engine and this text fed to servo controller helps in actuating the joints of identical robot arms. Cooperative manipulators are used for handling radioactive elements and also in the field of medicine as rehabilitation aid and also in surgeries. An Android app specifically built for this purpose communicates through Bluetooth technology makes the interface for end-user simple to control both identical robot arms simultaneously.
\end{abstract}

Keywords: Cooperative manipulators; Speech Recognition and Control; Android Application; Assistive manipulators;

\section{INTRODUCTION}

Coop ooperative manipulators[1] refers to the cooperation of two serial manipulators that physically interact with an object, apply forces on it to move or perform a specific task. They were introduced to replace dirty, difficult and dangerous jobs in manufacturing. Goertz constructed a cooperative manipulator in the 1940s for handling radioactive substances.

Dual-arm manipulators were considerably improved from then, yet its control is still conventional. Such Cooperative manipulators are challenging to control and hence, we define speech-based interfaces to control two identical robot arms.

Speech Recognition is a complex problem where string of words is associated to speech in contrast to the way where human recognize speech relating to concepts. In Automatic Speech Recognition, recognition is performed seeking the string of words that best match the acoustic observations according to the available model as shown in Figure 1. All variability in speech including

- Vocal Range (pitch and formant frequencies)

- Age, gender of the speaker

- Voice Quality

- Accent

Revised Manuscript Received on December 02, 2019

* Correspondence Author

N. Saravanan*, Division of Mechatronics, Department of Production Technology, Madras Institute of Technology, Anna University, Chennai-44, India. Email: saravanan_n@msn.com

Dr. R. Sivaramakrishnan, Department of Production Technology, Madras Institute of Technology, Anna University, Chennai-44, India. Email srk@mitindia.edu
- Emotional State

- Speech Style

Should be nullified to convert speech to text form.

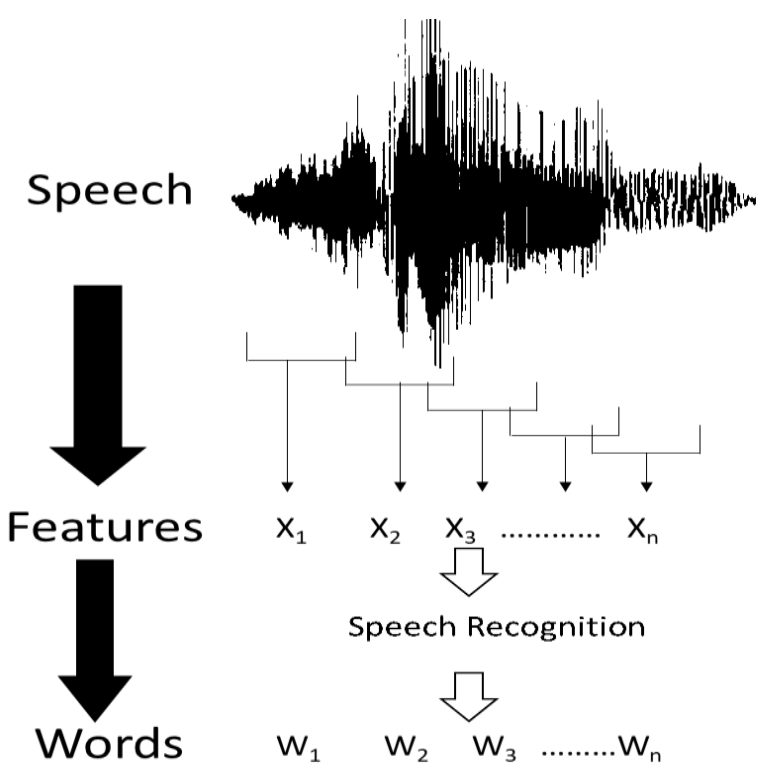

Figure 1 Conversion of Speech to text

Here, conversion of speech in Indian languages including English, Hindi and Tamil to string of words in corresponding language is done that frames in to sentences. These sentences could act as a control command for actuation of dual-arm Cooperative manipulators. The block diagram of speech-based control of two identical robot arms is as shown in Figure 2.

\section{DESIGN AND SELECTION OF TWO IDENTICAL ROBOT ARMS}

Any Two Robot Arms with Twisting-Revolute-Revolute with additional pitch and yaw Configuration would be applicable for cooperative operation assisting in surgical tasks or assistive rehabilitation aid[2]. Two identical robot arms from lynxmotion were selected and these assembled robot arms may be fitted into either side of a wheelchair for assistive aids or may be used in surgeries where two robot arms need to work in conjunction with one another. Two Kinova Jaco Spherical Robot arm can also be used for the same purpose. Kinematic Analysis of Robotic Arms[3] is done theoretically and programmatically to achieve consistency in point-to-point control of dual-arm robot arms. 


\section{Design and Control of Dual-Arm Cooperative Manipulator using Speech Commands}

Such dual-arm robots could be deployed and controlled in a remote way through existing wireless technologies for handling difficult jobs like cleaning the gutter, handling materials emanating heavy radiation and many more.

Here we use Statistical based Speech Recognition Models to convert sequence of speech over time to text. CMU Sphinx API[4] built using Hidden Markov Model recognizes short vocabulary speech in English, Hindi and Tamil.
Appropriate Language Models, Acoustic Models and Lexicon were generated for the above Indian languages and training using Baum-Welch Re-estimation procedures were done.

In Table 1 we describe the link lengths and base radius of both robot arms. Here in Figure 3, we present a pictorial representation of two identical robot manipulators grasping an object. Here in Figure 4, we present a CAD model of two identical robot arms acting in conjunction with one another.

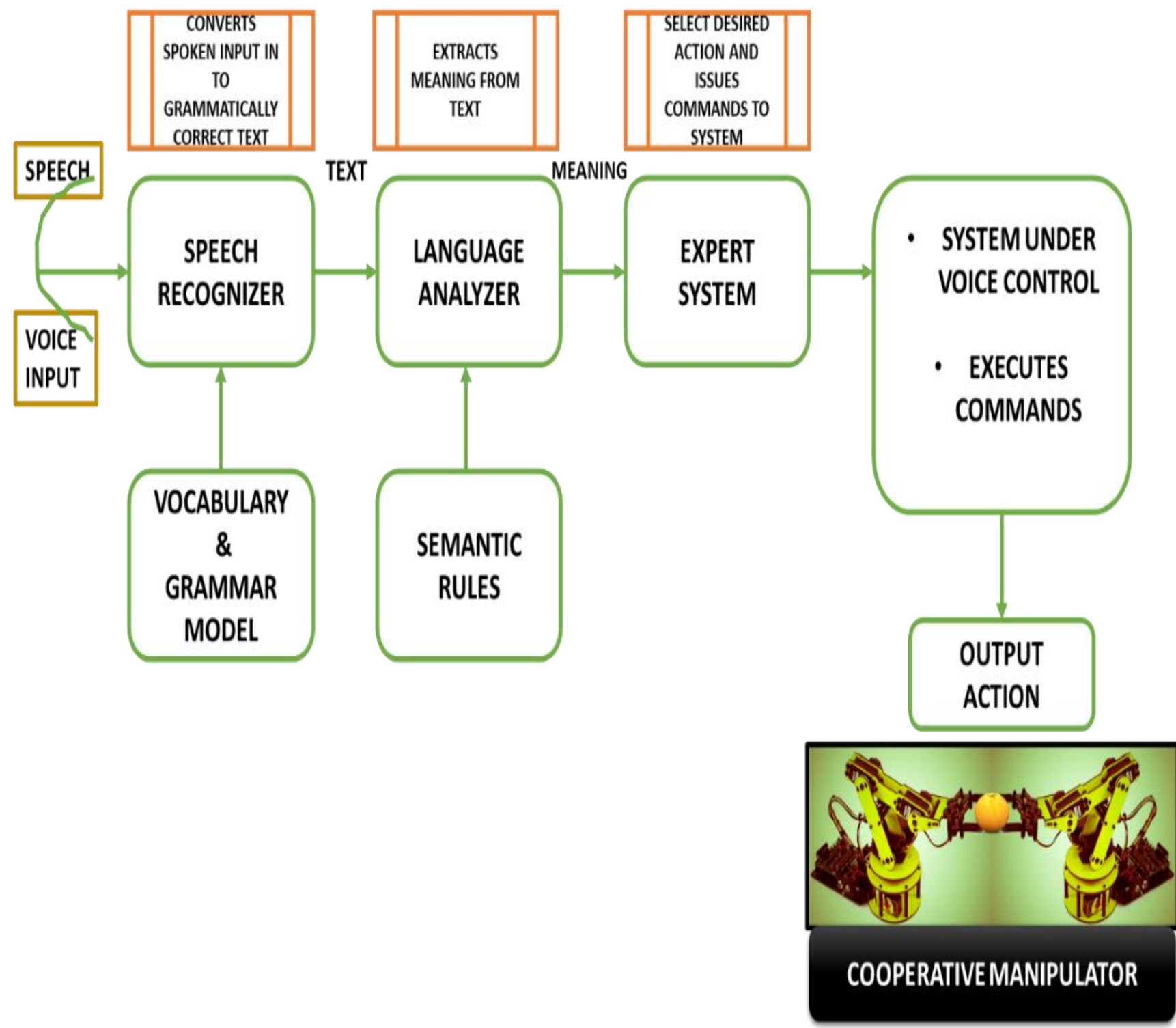

Figure 2 Block diagram of speech-based control of two identical robot arms

Table 1 Link lengths and base radius of both robot arms

\begin{tabular}{|l|l|l|l|l|l|l|}
\hline ROBOT-A & Inch & $\mathrm{Cm}$ & ROBOT-B & Inch & Cm \\
\hline Base Radius & 1.94 & 4.928 & 7.899 \\
\cline { 2 - 3 } Base height & 3.11 & Base Radius & 1.94 & 4.928 \\
\hline Forearm Length & 4.75 & 12.065 & Base height & 3.11 & 7.899 \\
\hline Arm Length & 4.75 & 12.065 & Forearm Length & 4.75 & 12.065 \\
\hline Hand Length & 5.61 & 14.249 & Arm Length & 4.75 & 12.065 \\
\cline { 2 - 4 } & Hand Length & 5.61 & 14.249 \\
\hline
\end{tabular}




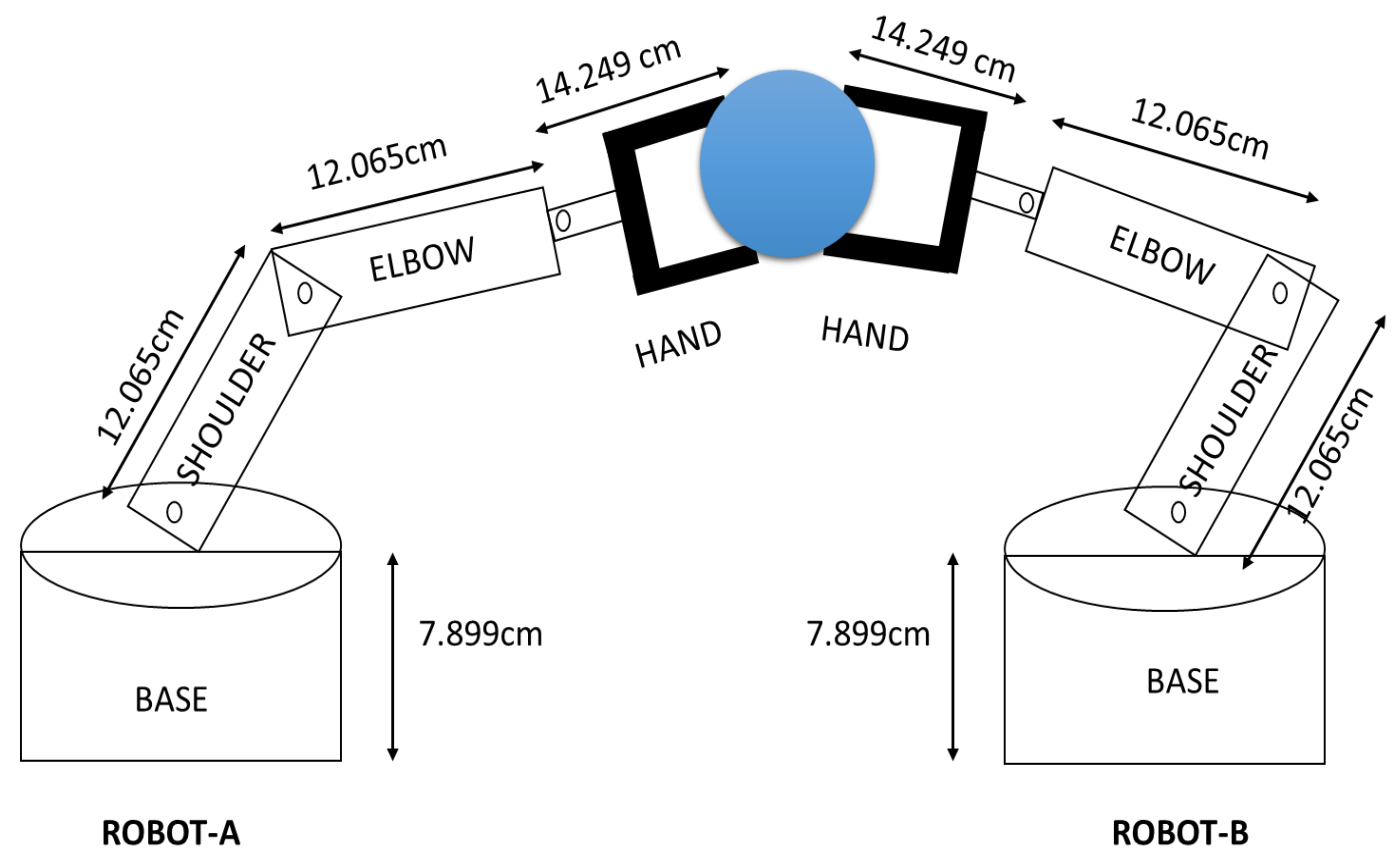

Figure 3 Two identical robot manipulators are grasping an object

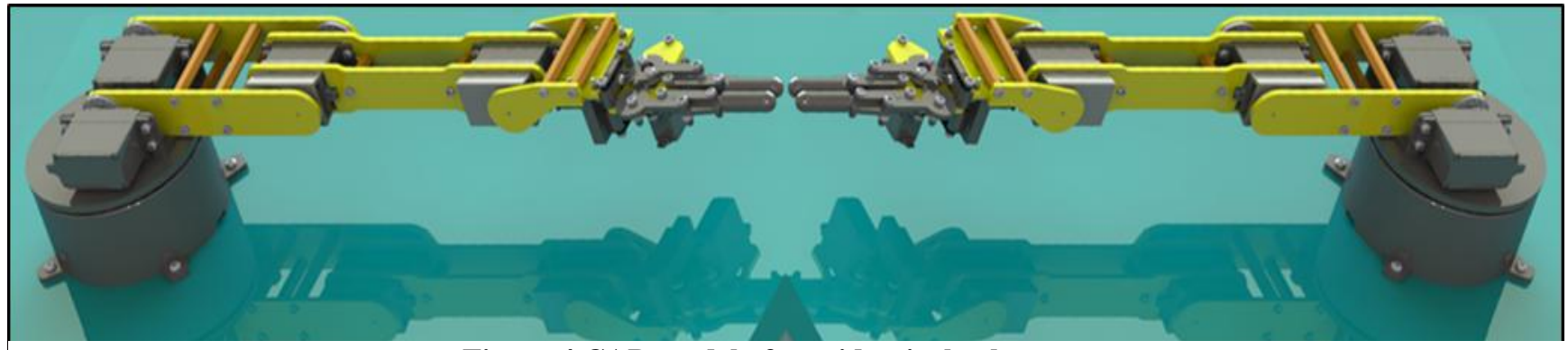

Figure 4 CAD model of two identical robot arms

\section{KINEMATIC ANALYSIS OF T-R-R CONFIGURATION ROBOT ARMS}

Kinematics[3] is the examination of the motion of robots. It can be Forward Kinematics, wherein Cartesian coordinates are found using Joint angles and Inverse Kinematics, wherein Joint space coordinates are found using Cartesian Coordinates.

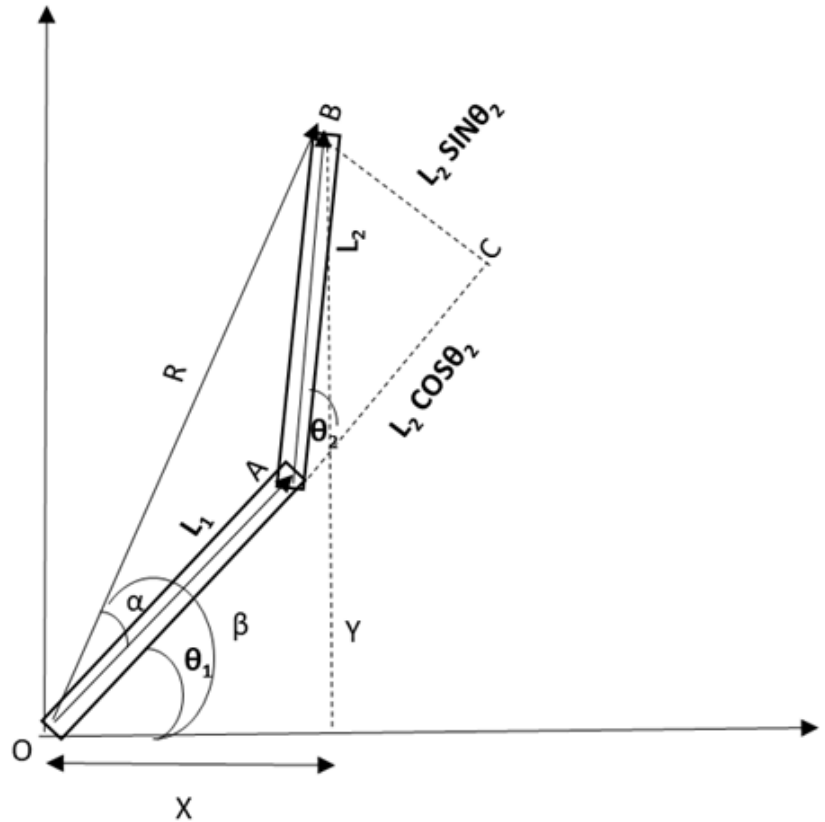

Figure 5. Schematic Representation of two degrees of Freedom R-R Planar Robot

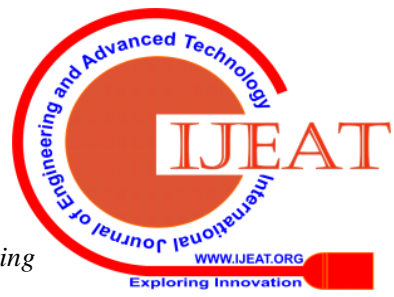


Design and Control of Dual-Arm Cooperative Manipulator using Speech Commands

\section{A. FORWARD KINEMATICS}

\section{A. FORWARD KINEMATICS:}

Forward Kinematic Equations are as shown in Table 2.

Table 2 Forward Kinematic Equations of T-R-R Configuration Robots

\begin{tabular}{|l|}
\hline \multicolumn{1}{|c|}{ FORWARD KINEMATICS } \\
\hline ROBOT-A \\
\hline$X=\left[L_{A 1} \cos \theta_{1}+L_{A 2} \cos \left(\theta_{1}+\theta_{2}\right)\right] \cos \emptyset$ \\
$Y=\left[L_{A 1} \operatorname{SIN} \theta_{1}+L_{A 2} \operatorname{SIN}\left(\theta_{1}+\theta_{2}\right)\right]$ \\
$Z=\left[L_{A 1} \cos \theta_{1}+L_{A 2} \cos \left(\theta_{1}+\theta_{2}\right)\right] \operatorname{sIN} \emptyset$ \\
\hline $\operatorname{ROBOT}-B$ \\
\hline$X=\left[L_{B 1} \cos \theta_{1}+L_{B 2} \cos \left(\theta_{1}+\theta_{2}\right)\right] \cos \emptyset$ \\
$Y=\left[L_{B 1} \operatorname{SIN} \theta_{1}+L_{B 2} \operatorname{SIN}\left(\theta_{1}+\theta_{2}\right)\right]$ \\
$Z=\left[L_{B 1} \cos \theta_{1}+L_{B 2} \cos \left(\theta_{1}+\theta_{2}\right)\right] \operatorname{SIN} \emptyset$ \\
\hline
\end{tabular}

\section{B. INVERSE KINEMATICS:}

To find the joint angles of T-R-R configuration manipulator, we perform inverse kinematics of two degrees of freedom R-R planar manipulator, since the manipulators we use are of the configuration R-R with twisting base.

\section{INVERSE KINEMATICS OF TWO DEGREE OF FREEDOM R-R PLANAR ROBOT:}

Here we consider Cartesian Coordinates $(\mathrm{r} 1=[\mathrm{X} 1, \mathrm{Y} 1], \mathrm{r} 2$ $=[\mathrm{X} 2, \mathrm{Y} 2])$ are known along with the Link Lengths. Here, we follow a geometric approach instead of D-H (Denavit Hartenberg) matrix approach. Finally, we arrive at all angles including $\theta_{1}, \theta_{2}$ (Joint angles) and $\varphi$ (rotation angle).

$$
\begin{aligned}
& B C=L_{2} \operatorname{SIN} \theta_{2}(1) \\
& O C=L_{1}+L_{2} \operatorname{Cos} \theta_{2}(2) \\
& A C=L_{2} \operatorname{CoS} \theta_{2}(3) \\
& \tan \alpha=\frac{B C}{O C}=\frac{\mathrm{L} 2 \operatorname{SIN} \theta 2}{\mathrm{~L} 1+\mathrm{L} 2 \operatorname{Cos} \theta 2}
\end{aligned}
$$$$
\tan \beta=\frac{Y}{X}(5)
$$$$
r_{1}=\left[X_{1}, Y_{1}\right]=\left[L_{1} \operatorname{Cos} \theta_{1}, L_{1} \operatorname{SIN} \theta_{1}\right](6)
$$$$
r_{2}=\left[X_{2}, Y_{2}\right]=\left[\begin{array}{c}
L_{2} \operatorname{Cos}\left(\theta_{1}+\theta_{2}\right), \\
L_{2} \operatorname{SIN}\left(\theta_{1}+\theta_{2}\right)
\end{array}\right](7)
$$$$
X=L_{1} \cos \theta_{1}+L_{2} \cos \left(\theta_{1}+\theta_{2}\right)(8)
$$$$
Y=L_{1} \operatorname{SIN} \theta_{1}+L_{2} \operatorname{SIN}\left(\theta_{1}+\theta_{2}\right)(9)
$$$$
X^{2}+Y^{2}=L_{1}^{2} \cos ^{2} \theta_{1}+L_{2}^{2} \cos ^{2}\left(\theta_{1}+\theta_{2}\right)
$$$$
+2 L_{1} L_{2} \cos \theta_{1} \cos \left(\theta_{1}+\theta_{2}\right)
$$$$
+L_{1}^{2} \operatorname{SIN}^{2} \theta_{1}+L_{2}^{2} \operatorname{SIN}^{2}\left(\theta_{1}+\theta_{2}\right)+
$$$$
2 L_{1} L_{2} \operatorname{SIN} \theta_{1} \operatorname{SIN}\left(\theta_{1}+\theta_{2}\right)(10)
$$$$
X^{2}+Y^{2}=L_{1}^{2}+L_{2}^{2}+
$$$$
2 L_{1} L_{2} \cos \left(\theta_{1}-\theta_{1}-\theta_{2}\right)(11)
$$

Since,

$$
\cos ^{2} \theta+\operatorname{SIN}^{2} \theta=1
$$

and

$$
\begin{aligned}
& \cos (A-B)=\cos (A) \cos (B)+\operatorname{SIN}(A) \sin (B) \\
& X^{2}+Y^{2}=L_{1}^{2}+L_{2}^{2}+2 L_{1} L_{2} \cos \left(\theta_{2}\right)(12)
\end{aligned}
$$

Since

$$
\begin{aligned}
& \cos (-X)=\cos (X) \\
& \cos \theta_{2}=\frac{X^{2}+Y^{2}-L_{1}^{2}-L_{2}^{2}}{2 L_{1} L_{2}} \\
& \theta_{2}=\operatorname{atan} 2\left(\sin \theta_{2}, \cos \theta_{2}\right)
\end{aligned}
$$

$$
\theta_{2}=\operatorname{atan} 2\left( \pm \sqrt{1-\cos ^{2} \theta_{2}}, \cos \theta_{2}\right)
$$

The final equation for $\theta_{2}$ is

$\theta_{2}=\operatorname{atan} 2\left( \pm \sqrt{1-\left(\frac{X^{2}+Y^{2}-L_{1}^{2}-L_{2}^{2}}{2 L_{1} L_{2}}\right)}, \frac{X^{2}+Y^{2}-L_{1}^{2}-L_{2}^{2}}{2 L_{1} L_{2}}\right)$

Also,

$$
\beta-\alpha=\theta_{1}(16)
$$

$$
\begin{aligned}
\tan (\beta-\alpha)=\tan \theta_{1}(17) \\
\tan \theta_{1}=\frac{\tan \beta-\tan \alpha}{1+\tan \beta \tan \alpha}(18) \\
\tan \theta_{1}=\frac{\frac{Y}{X}-\frac{L_{2} \operatorname{SIN} \theta_{2}}{L_{1}+L_{2} \operatorname{Cos} \theta_{2}}}{1+\frac{Y}{X} \frac{L_{2} \operatorname{SIN} \theta_{2}}{L_{1}+L_{2} \operatorname{Cos} \theta_{2}}}
\end{aligned}
$$

$\tan \theta_{1}=\frac{Y\left[L_{1}+L_{2} \cos \theta_{2}\right]-X\left[L_{2} \operatorname{SIN} \theta_{2}\right]}{X\left[L_{1}+L_{2} \cos \theta_{2}\right]+Y\left[L_{2} \operatorname{SIN} \theta_{2}\right]}$

The final equation for $\theta_{1}$ is

Where,

$$
\theta_{1}=\operatorname{atan} 2(y, x)-\operatorname{atan} 2\left(Z_{2}, Z_{1}\right)
$$

$Z_{1}=L_{1}+L_{2} \cos \theta_{2}$ (21)

$Z_{2}=L_{2} \operatorname{SIN} \theta_{2}(22)$

\section{INVERSE KINEMATICS OF LYNXMOTION ROBOT ARM:}

Now, Robotic ARM can be represented as a $2 \mathrm{R}$ manipulator with a rotating base.

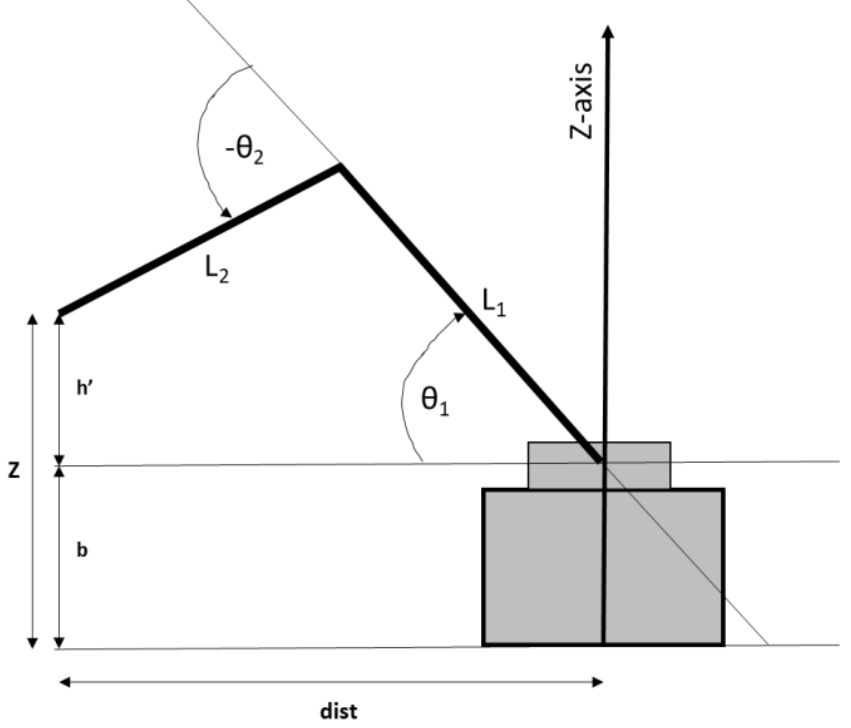

Figure 6 Side view of a TRR robot arm without the gripper 


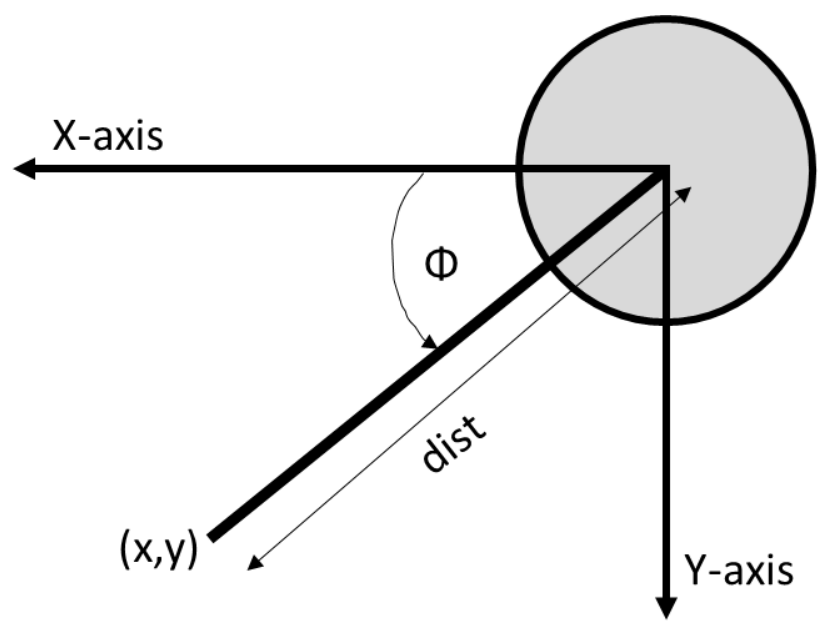

We can calculate the angles $\varphi, \theta_{1}, \theta_{2}$ from the position $(\mathrm{x}, \mathrm{y}, \mathrm{z})$ of the wrist.

$$
\varphi=\operatorname{atan} 2(y, x)
$$

To calculate angles $\theta_{1}$ and $\theta_{2}$, we first find the distance dist of the point $(\mathrm{x}, \mathrm{y})$ from origin $\mathrm{o}$ and we also determine the height above the shoulder joint.

$$
\begin{aligned}
& \text { dist }=\sqrt{x^{2}+y^{2}}(23) \\
& h^{\prime}=Z-b(24)
\end{aligned}
$$

Now, substitute $\mathrm{x}=$ dist and $\mathrm{y}=\mathrm{h}$ ' in the equations where we find $\theta_{2}$ and $\theta_{1}$ for $\mathrm{R}-\mathrm{R}$ planar manipulator. Kinematic Equations helped in determining the current state of the lynxmotion robot arm. However, our project aims in high level control interface using Speech commands.

\section{DESIGN OF SPEECH-BASED INTERFACE FOR CONTROL OF DUAL-ARM MANIPULATORS}

The text and speech corpora for control of dual-arm manipulators was created in Indic languages naming English, Hindi, and Tamil[4]. Here we use an offline speech recognition engine[5] so that control could be made wirelessly through smartphone and also doesn't require an active internet connection. The following Figure 10 shows Text and speech corpora created for control of cooperative manipulator. Language model, Acoustic model, and dictionary were created for languages English, Hindi, and Tamil so that end-user comfortable in these languages will be able to control two identical robot arms simultaneously. Hidden Markov Model forms the basis of my Recognition Engine where in The Forward Algorithm is used for Scoring, Viterbi Algorithm for Matching and Baum-Welch Learning algorithm for training. Speech recognition engine developed converts the above commands to text form, fed to servo controller for appropriate actuation of the robot[6].

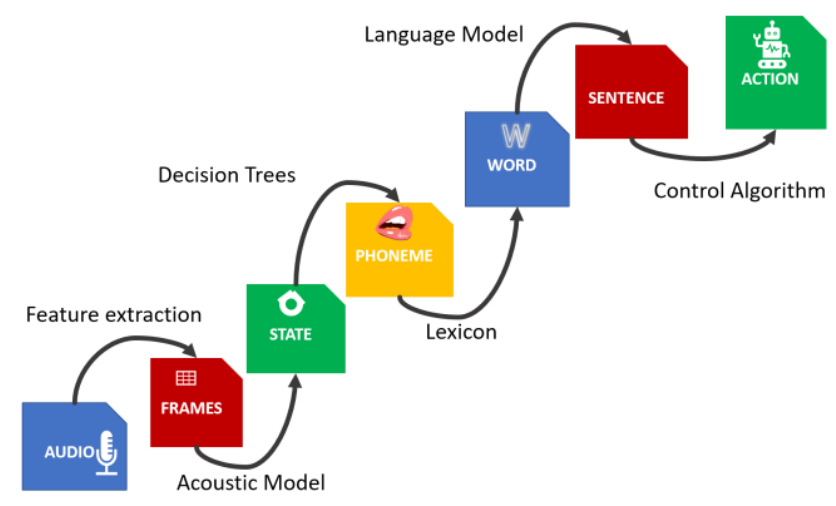

Figure 8 Speech to dual-arm robot actuation

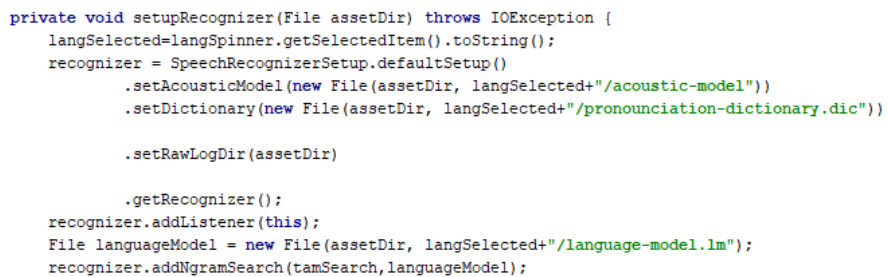

Figure 9 Code to set the Decoder

\section{SPEECH COMMANDS TO CONTROL COOPERATIVE MANIPULATOR}

\section{ENGLISH}

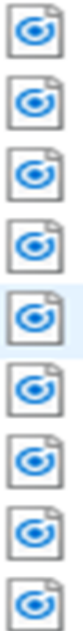

file_1
file_2
file_3
file_4
file_5
file_6
file_7
file_8
file_9

MOVE SHOULDER UP

MOVE SHOULDER DOWN

MOVE ELBOW UP

MOVE ELBOW DOWN

MOVE WRIST UP

MOVE WRIST DOWN

OPEN GRIPPER

CLOSE GRIPPER

ROTATE BASE

\section{TAMIL}

தோவை உயர்த்து

தோவை இறக்கு

முழங்கை உயர்த்து

முழங்கை இறக்கு

மணிகட்டை உயர்த்து

மணிகட்டை இறக்கு

விரலை தற

விரலை யூ (b)

அடித்தலத்தை சுற்று
HINDI

कंधे को ऊपर करें।

कंधे को नीचे करें।

कोहनी को ऊपर करें ।

कोहनी को नीचे करें।

कलाई को ऊपर करें।

कलाई को नीचे करें।

उंगली खोलें।

उंगली बंद करें।

बेस घुमाएँ।

Figure 10 Speech Commands to Control Cooperative Manipulator

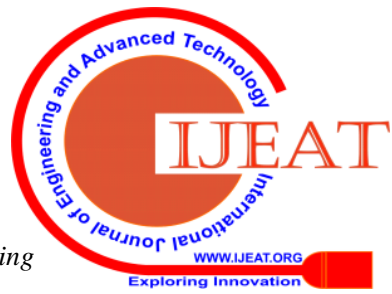




\section{Design and Control of Dual-Arm Cooperative Manipulator using Speech Commands}

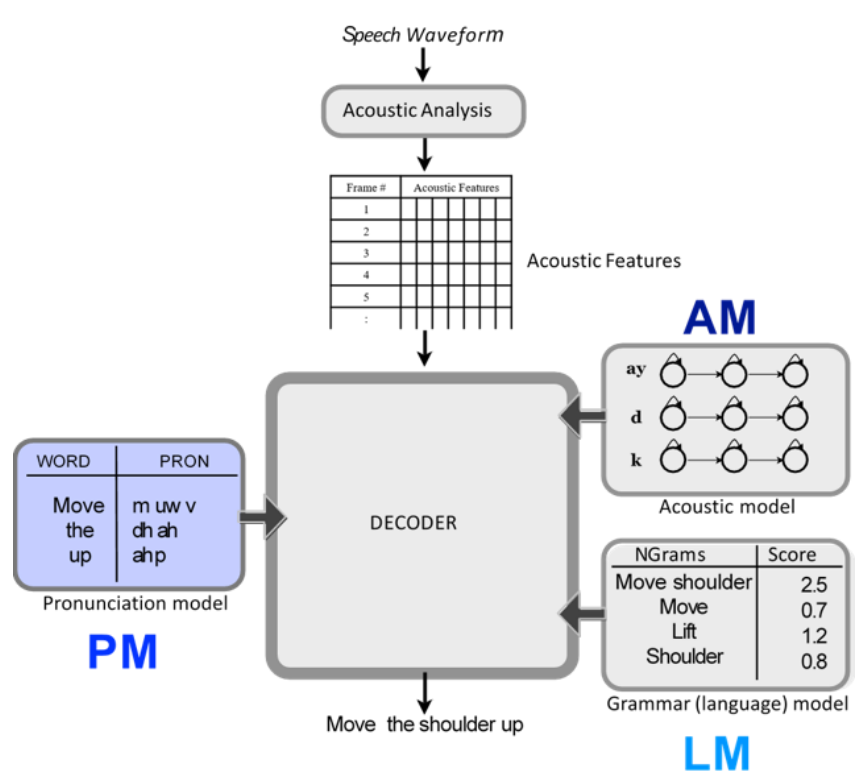

Figure 11 Block Diagram of Decoder

\section{IMPLEMENTATION OF CONTROL USING ANDROID APPLICATION}

We develop an android application[7] using HC-05 Bluetooth technology coupled with Arduino UNO and servo controller. Two identical serial arms can be controlled through speech commands said through the microphone of a smartphone which is then converted into text form through the mobile app and after processing the language, the mobile app sends appropriate commands serially to Arduino board, which then sends signals to motor controller for simultaneous actuation of servo motors. The joints of ROBOT-A are connected to the channels 1 through 4 and the joints of ROBOT-B is connected to the channels 26 through 30 .

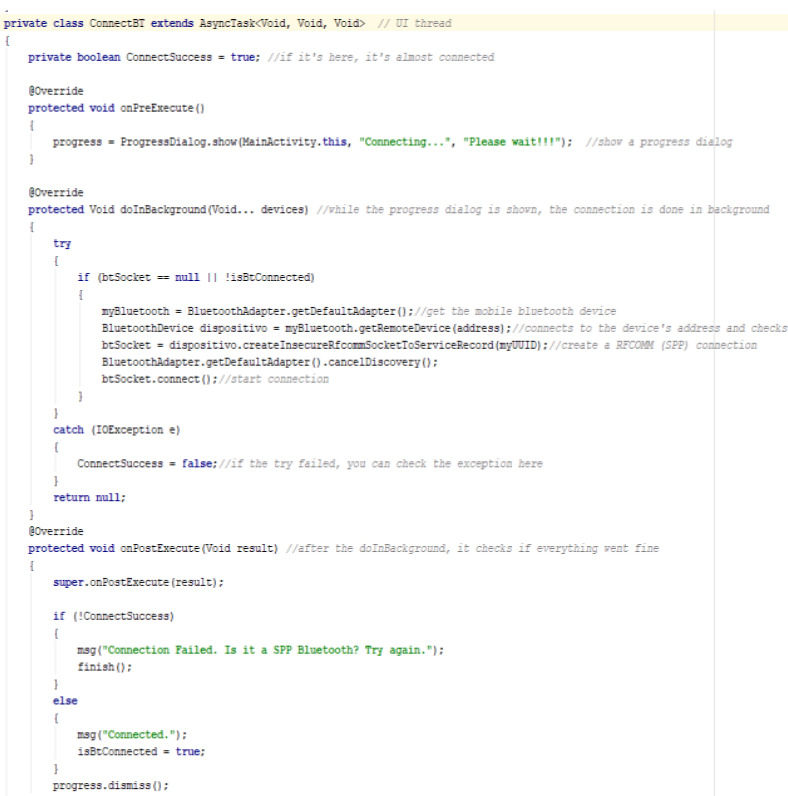

Figure 12 Android code to connect to Bluetooth

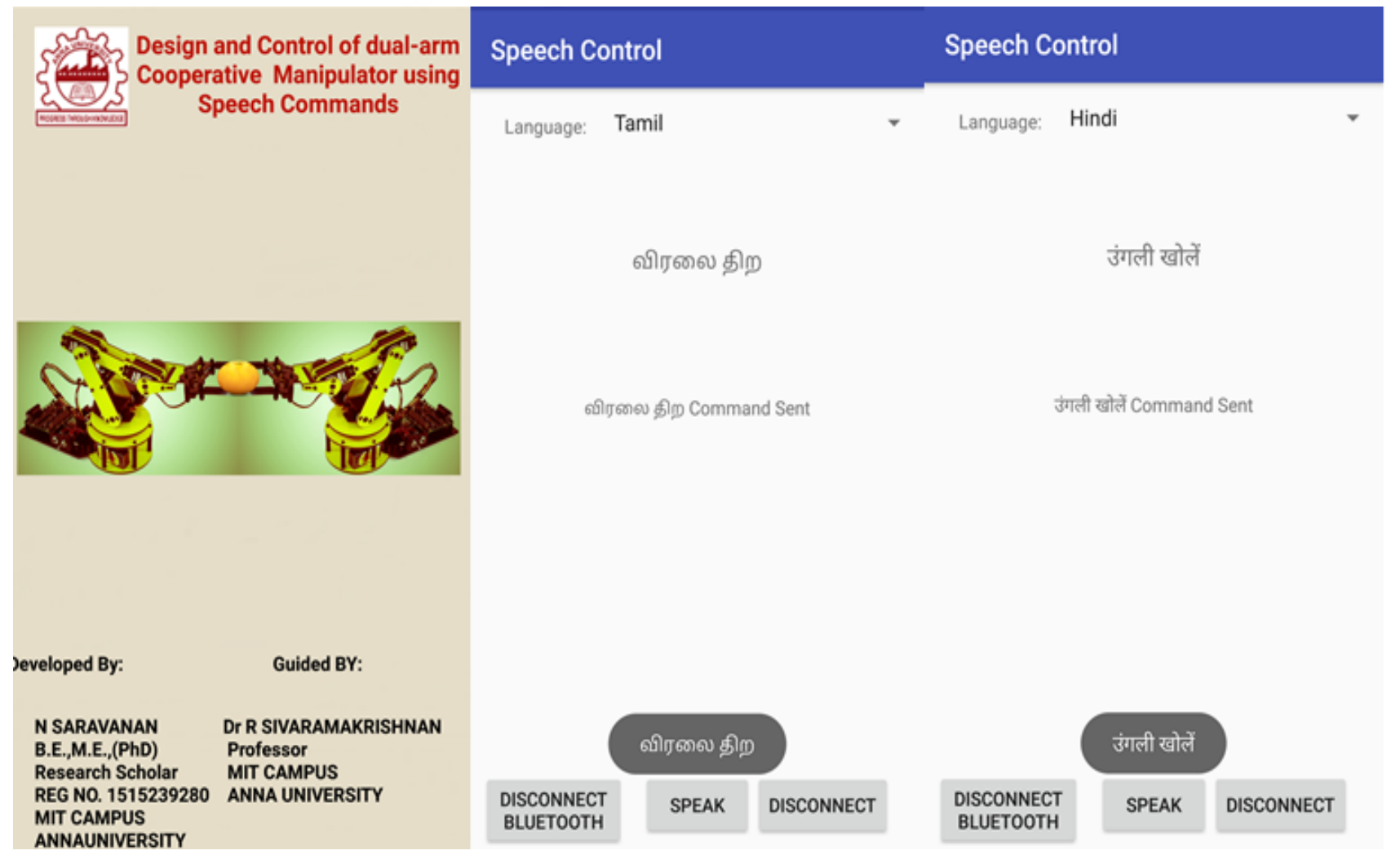

Figure 13. Sample Android Application for control of dual arm manipulators through speech 


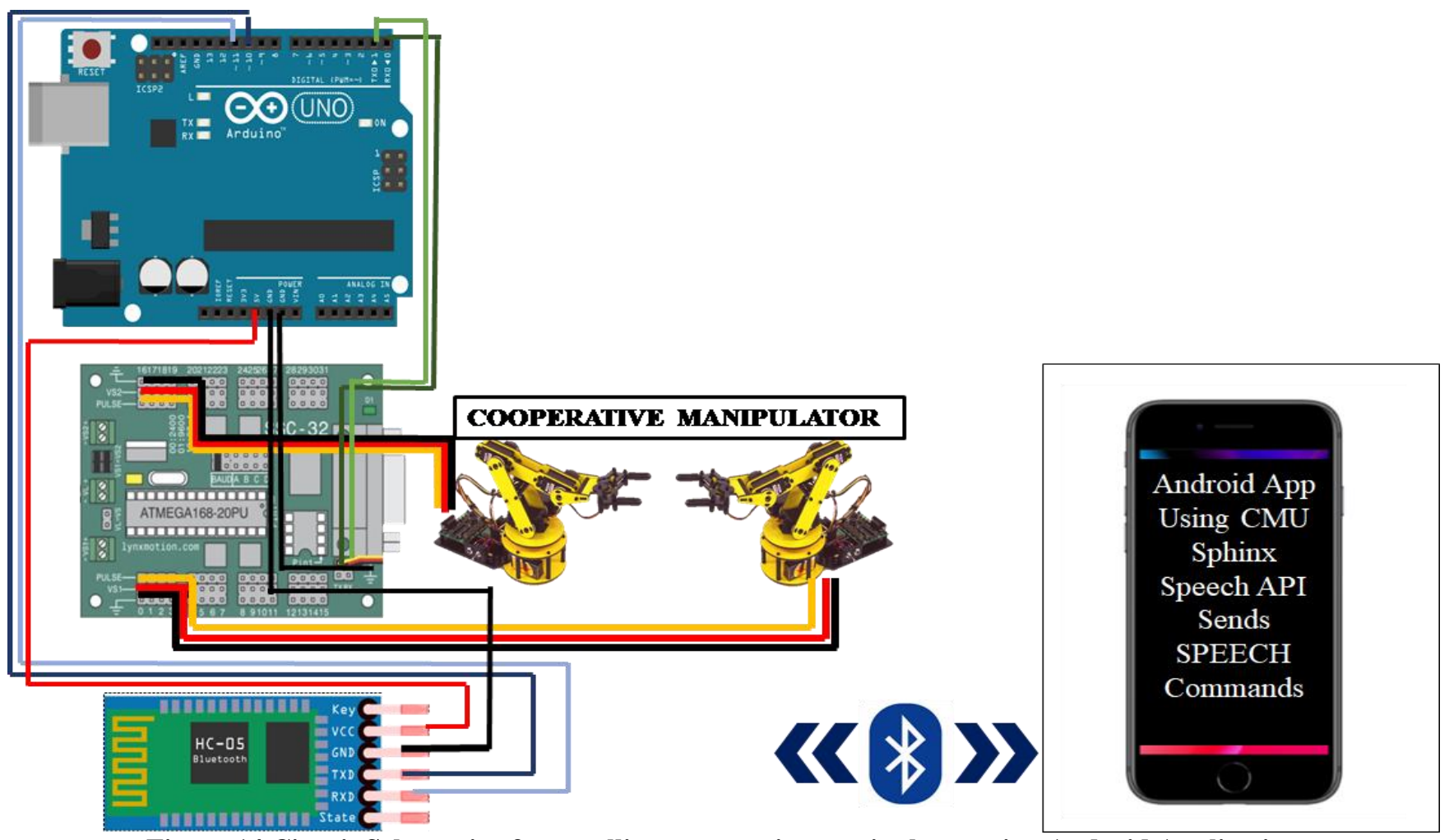

Figure 14 Circuit Schematic of controlling cooperative manipulator using Android Application

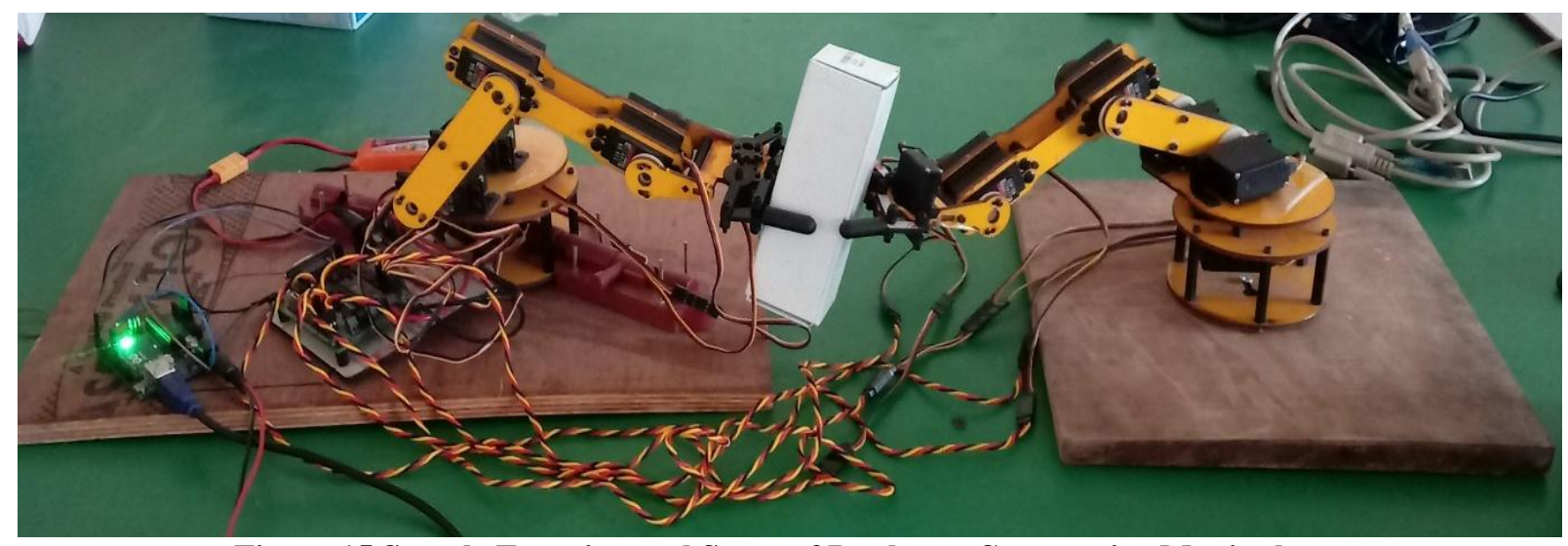

Figure 15 Sample Experimental Setup of Dual arm Cooperative Manipulator

Table 3 Experimental Results- Speech-Based Control of Cooperative Manipulator

\begin{tabular}{|c|c|c|c|c|c|c|c|c|c|}
\hline \multirow[t]{2}{*}{ Gender } & \multicolumn{3}{|c|}{$\begin{array}{l}\text { Total Number of Robot } \\
\text { Commands Spoken } \\
\text { (184) }\end{array}$} & \multicolumn{3}{|c|}{$\begin{array}{l}\text { Total Number of Robot } \\
\text { Commands Recognized by } \\
\text { Robot-A and Robot-B } \\
\text { (178) }\end{array}$} & \multicolumn{3}{|c|}{$\begin{array}{l}\text { Total Number of Robot } \\
\text { Commands went Unrecognized } \\
\text { by Robot-A and Robot-B } \\
\text { (6) }\end{array}$} \\
\hline & English & Hindi & Tamil & English & Hindi & Tamil & English & Hindi & Tamil \\
\hline Male & 40 & 40 & 42 & 39 & 39 & 42 & 1 & 1 & 0 \\
\hline Female & 20 & 20 & 22 & 19 & 19 & 20 & 1 & 1 & 2 \\
\hline
\end{tabular}

\section{RESULT AND DISCUSSION}

The designed Cooperative Manipulator[8] was checked for its movements for individual robot commands for Point-To-Point Control through Speech. Candidates from both genders were allowed to speak and actuation of cooperative manipulator was monitored. Usually, Cooperative manipulators are controlled through Teach
Pendant or require a complex mechanism. Here we just provide a smartphone to the end-user who just speaks to control and thus that could be an assistive aid if both robots were deployed on either side of the wheel chair. The prototype was tested and had an accuracy of $96.73 \%$.

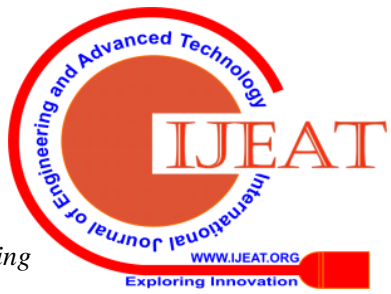




\section{Design and Control of Dual-Arm Cooperative Manipulator using Speech Commands}

The following figure shows the criterion tested for Actuation of Robot-A and Robot-B.

\begin{tabular}{|l|l|l|}
\hline Criterion & $\begin{array}{l}\text { Recognition of } \\
\text { Robot Command }\end{array}$ & $\begin{array}{l}\text { Actuation of } \\
\text { Robot-A and } \\
\text { Robot-B }\end{array}$ \\
\hline True Positives & $\begin{array}{l}\text { Correctly } \\
\text { Recognized }\end{array}$ & Correctly Actuated \\
\hline $\begin{array}{l}\text { True } \\
\text { Negatives }\end{array}$ & $\begin{array}{l}\text { Correctly } \\
\text { Recognized }\end{array}$ & $\begin{array}{l}\text { But Incorrectly } \\
\text { Actuated or } \\
\text { Actuation NOT } \\
\text { DONE }\end{array}$ \\
\hline $\begin{array}{l}\text { False } \\
\text { Positives }\end{array}$ & $\begin{array}{l}\text { Incorrectly } \\
\text { Recognized }\end{array}$ & $\begin{array}{l}\text { But Actuation } \\
\text { Done }\end{array}$ \\
\hline $\begin{array}{l}\text { False } \\
\text { Negatives }\end{array}$ & $\begin{array}{l}\text { Incorrectly } \\
\text { Recognized }\end{array}$ & $\begin{array}{l}\text { And Actuation } \\
\text { NOT DONE }\end{array}$ \\
\hline
\end{tabular}

Figure 16 Criterion Tested based on Recognition and Actuation

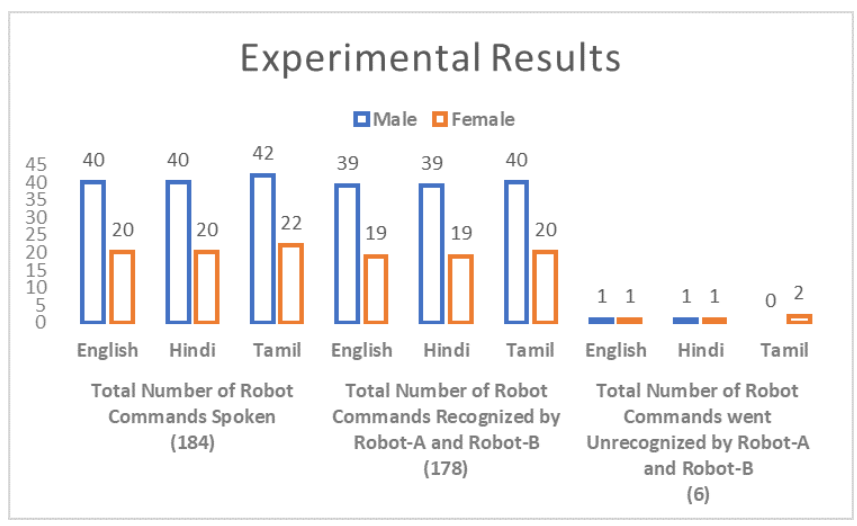

Figure 17 Experimental Results-Actuation on Recognition

\section{CONCLUSION}

There were no or minimal False Positives and True Negatives concluding our control of cooperative manipulator to be more robust. The Training Mode of Speech Recognition[9] had the following Phases and it listed only minimal word error rate encouraging us to develop a robust system for control of two identical lynxmotion robot arms. Cooperative manipulators[6] designed and controlled through speech could be deployed in assisting Surgeries[2], or manipulating radioactive elements simultaneously or combinedly operating on the object twice its payload for pick-and-place[10].

\section{REFERENCES}

1. Caccavale, F., Cooperative Manipulators, in Encyclopedia of Systems and Control, J. Baillieul and T. Samad, Editors. 2015, Springer London: London. p. 230-235.

2. White, K.S., Speech recognition implementation in radiology. Pediatric radiology, 2005. 35(9): p. 841-846.

3. $\mathrm{Hu}, \mathrm{B} ., \mathrm{B}$. Li, and H. Cui, Design and kinematics analysis of a novel serial-parallel kinematic machine. Proceedings of the Institution of Mechanical Engineers, Part C: Journal of Mechanical Engineering Science, 2016. 230(18): p. 3331-3346.

4. Hyassat, H. and R. Abu Zitar, Arabic speech recognition using SPHINX engine. International Journal of Speech Technology, 2006. 9(3): p. 133-150.
5. Saraswathi, S. and T.V. Geetha. Improvement in Performance of Tamil Phoneme Recognition using Variable Length and Hybrid Language Models. in 2007 International Conference on Signal Processing, Communications and Networking. 2007.

6. Lei, Z., et al. Artificial robot navigation based on gesture and speech recognition. in Proceedings 2014 IEEE International Conference on Security, Pattern Analysis, and Cybernetics (SPAC). 2014. IEEE.

7. Gandhewar, N., R.J.I.J.o.C.S. Sheikh, and Engineering, Google Android: An emerging software platform for mobile devices. 2010. 1(1): p. 12-17.

8. Wen, J.T. and K. Kreutz. Motion and force control for multiple cooperative manipulators. in Proceedings, 1989 International Conference on Robotics and Automation. 1989. IEEE.

9. Shashidhar, G., K. Koolagudi, and R. Sreenivasa, Emotion recognition from speech: a review. Springer Science+ Business Media, 2012. 15: p. 99-117.

10. Caccavale, F., et al., Six-dof impedance control of dual-arm cooperative manipulators. IEEE/ASME Transactions On Mechatronics, 2008. 13(5): p. 576-586.

\section{AUTHORS PROFILE}

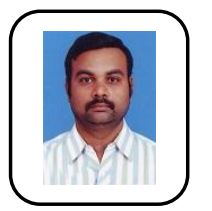

Saravanan N. is a Computer Engineer mastered in Mechatronics Engineering and currently pursuing his research in the field of Speech Recognition and Control for Industrial Robots. His Research interests are in the field of Bio-mechatronics, Machine Learning algorithms and many more.

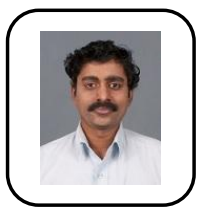

Dr R. Sivaramakrishnan, is a Mechanical Engineer whose research areas include Parallel Manipulators, Industrial Robotics, Material Science, Image Processing and many more. He is Professor and Head of the Department of Production Technology and has produced numerous doctorates under his guidance. 